\title{
Un ensayo controlado de
}

\section{intervención social en familias de pacientes esquizofrénicos*}

\section{J. P. Leff, L. Kuipers, R. Berkowitz, R. Eberlein-Vries y D. Sturgeon}

Se ha establecido una intensa relación entre el nivel de emoción expresada (EE) mostrada por familiares y el pronóstico de la esquizofrenia en pacientes que viven con ellos (Brown et al., 1962, 1972; Vaughn y Leff, 1976a; Leff y Vaughn, 1981). La recaída en la esquizofrenia es más probable si los pacientes viven con familiares excesivamente críticos y/o con una implicación emocional excesiva. Tales familiares son considerados, de EE alta. Dos factores parecen proteger a los pacientes que viven con familiares de EE alta: mantenimiento regular de la terapia con medicamentos neurolépticos y el establecimiento de una distancia social entre el paciente y sus familiares, medida por el contacto directo durante una semana típica. Parece, además, que estos dos factores son aditivos en su efecto protector. La evidencia de estas conclusiones se deriva del conjunto de datos aportados por los dos estudios más recientes (Brown et al, 1972; Vaughn y Leff (1976a). Reproducimos aquí el cuadro en que se hacen patentes estos resultados.

Fig. 1-Porcentajes de recaida, durante nueve meses, en un grupo total de 128 pacientes esquizofrénicos

\begin{tabular}{|c|c|c|c|c|}
\hline \multirow{8}{*}{$\begin{array}{c}\text { Grupo } \\
\text { total }\end{array}$} & \multirow{2}{*}{ EE baja $13 \%$} & & \multicolumn{2}{|l|}{ Subgrupos } \\
\hline & & & $\int 1$. Con medicación & $12 \%$ \\
\hline & \multicolumn{4}{|c|}{ Contacto directo } \\
\hline & \multirow{4}{*}{ EE alta $51 \%$} & $<35$ hrs. $28 \%$ & \{ 3. Con medicación & $15 \%$ \\
\hline & & & 4. Sin medicación & $42 \%$ \\
\hline & & & $\Omega^{\circ}$ & \\
\hline & & $>35$ hrs. & 6. Sin medicación & $92 \%$ \\
\hline & & & & \\
\hline
\end{tabular}

El problema de la dirección de la causalidad en la relación entre la EE de los familiares y la recaída de los pacientes ha sido estudiado utilizando técnicas estadísticas para definir la función de los.posibles factores mediadores, tales como la alteración conductual de los pacientes. Se eliminó la posibilidad de que tal conducta pudiera llevar tanto a una EE alta de los familiares como a la recaída de los pacientes merced al uso de una amplia

* Publicado originalmente en British Journal of Psychiatry, 1982, 141, 121-134. 
variedad de tratamientos estadísticos (Brown et al., 1972; Vaughn y Leff, 1976a). No obstante, la relación causal entre la $\mathrm{EE}$ alta y la recaída de pacientes esquizofrénicos y/o la que existiría entre una $\mathrm{EE}$ baja y el buen estado de los pacientes, sólo puede demostrarse convincentemente a través de un enfoque experimental en el que se manipule la EE de los familiares y se compruebe la tasa de recaída de los pacientes. Idéntico argumento es aplicable a la posible naturaleza protectora de unrcontacto directo reducido, que únicamente puede establecerse, sin ningún género de dudas, a través de un diseño experimental. El presente estudio presenta un experimento dirigido a estos dos objetivos.

\section{METODO}

\section{Diseño}

Leff (1981) ha explorado con detalle los problemas que plantea todo ensayo controlado de cualquier terapia social. Los diseños de doble ciego con placebo que se utilizan en ensayos controlados de medicación constituyen buen punto de partida, si bien es necesario introducir algunas modificaciones dada la naturaleza de los tratamientos sociales. Por ejemplo, no se pueden establecer placebos para un tratamiento social. Toda interacción entre el paciente y un miembro del equipo, aunque sea insignificante, tiene un potencial terapéutico, incluso un procedimiento de evaluación estandarizado. Reconocemos que no existe una solución ideal para todos los problemas inherentes; pero consideramos que nuestro diseño es el óptimo en las actuales circunstancias.

Optamos por estudiar pacientes con un alto riesgo de recaída, constituidos por el subgrupo 6 de la figura 1. Virtualmente, todos estos pacientes, que no tomaban medicación de modo regular y que vivían en contacto directo con familiares de $\mathrm{EE}$ alta, recayeron en el transcurso de nueve meses. Dada la probada eficacia de la administración continuada de neurolépticos para prevenir las recaídas en la esquizofrenia (Leff y Wing, 1971; Hogarty y Goldberg, 1973), no consideramos ético suspender la medicación a tales pacientes con este alto nivel de riesgo. Nuestra estrategia consistió en asegurar que todos.los pacientes objeto del estudio continuaran tomando neurolépticos en forma de inyecciones de acción prolongada, para evitar problemas de cooperación. Los pacientes se distribuyeron, al azar, en un grupo experimental, que fue objeto de la intervención social, y un grupo de control, que recibió los cuidados habituales de los pacientes externos. Al planificar nuestro diseño nos vimos enfrentados con la decisión de administar un tratamiento social no específico al grupo de control o permitir a sus responsables clínicos que les tratasen conforme a su práctica usual. Ambos procedimientos presentaban sus inconvenientes y optamos por el último, pensando que tendríamos que establecer que las experiencias de tratamiento de las familias del grupo experimental y el de control eran sustancialmente diferentes (Orford y Edwards, 1977).

\section{El paquete de intervenciones sociales}

En la figura 1 puede apreciarse que, a condición de si el bajo contacto 
ción de recaídas del 53 por 100 al 15 por 100 . En términos del efecto sobre la tasa de recaídas, ello equivaldría a alterar el nivel de EE de los familiares pasando los de alta EE a baja (compárense los subgrupos 1,2 y 3). De ahí que los objetivos de la intervención social fueron reducir el contacto por debajo del nivel crucial de 35 horas por semana (Brown et al., 1972) y/o reducir la EE de los familiares. Less (1976) estableció una gama de estrategias con las que podría esperarse la consecución de estos objetivos. Un posible diseño para nuestro estudio consistía en asignar, al azar, familias experimentales a una serie de intervenciones sociales, por ejemplo, un programa educacional, sesiones familiares o modificación de conducta. Estimamos que este proyecto era demasiado ambicioso en términos del número de sujetos que requería y, en su lugar, compilamos un paquete de intervenciones sociales, que debía aplicarse de modo flexible de acuerdo con las necesidades de la familia. Así, empezamos a comparar la relativa efectividad de este paquete con los cuidados habituales que reciben pacientes externos, con la expectativa de que si se mostraba superior los elementos individuales podrían evaluarse por separado en un estudio posterior.

La intervención consta, básicamente, de tres elementos: un programa educativo, un grupo de familiares y sesiones familiares.

\section{Programa educativo}

Consistió en cuatro lecturas sobre etiología, síntomas, desarrollo, tratamiento y manejo de la esquizofrenia. Meditamos largamente la redacción de estas lecturas, que reflejaban el consenso de nuestras opiniones sobre tales temas, en un lenguaje coloquial, evitando la jerga de modo estricto. Estas lecturas se leían, en voz alta, a cada familia en su casa. Inicialmente se realizaron cuatro visitas, una para cada tema; pero, tras la experiencia con algunas familias, decidimos que sería preferible dar dos lecturas a la vez. Después de cada lectura, concedíamos un tiempo ilimitado para que los familiares formularan sus preguntas.

De hecho, este elemento del paquete se evaluó por separado mediante la Entrevista de Conocimiento (Knowledge Interview) administrada antes y después del programa educacional. Esto queda expuesto, en detalle, en el epigrafe «Evaluación».

\section{El grupo de familiares}

Inicialmente se concibió como un medio de reunir familiares de alta y baja EE. Además de los estudios de emoción familiar, contábamos con la evidencia de estudios psicofisiológicos (Tarriet et al., 1979; Sturgeon $e t$ al., 1981), en los que se pone de manifiesto que los familiares con EE baja habían encontrado los medios de afrontar los problemas diarios de convivencia con pacientes esquizofrénicos, no solamente evitando afectar negativamente a los pacientes sino prestándoles un apoyo activo.

Nuestro propósito fue utilizar el grupo como medio de alterar los estilos de enfrentamiento de los familiares con $\mathrm{EE}$ alta, de manera que llegasen a adoptar pautas semejantes a la de los familiares de EE baja. Conocíamos algunos de los problemas que se planteaban a los familiares por el estudio de Creer y Wing (1975) e igualmente fueron valiosas las experiencias de Priestley con un grupo similar de familiares (Priestley, 1979). Con esta información estructuramos el grupo de manera que los terapeu- 
tas actuaran como facilitadores. Se animó a los familiares, tanto de alta como de baja EE., a que presentasen sus problemas y soluciones en la reunión y a que los compartieran con los demás que estaban en situación similar. El objetivo era facilitarles el aprendizaje de estrategias de enfrentamiento que les eran desconocidas y, finalmente, ayudarles a que adoptasen un enfoque diferente en sus casas. Así, el grupo abordaba dificultades, reales o potenciales, experimentadas por los familiares, dejando en un segundo plano la interpretación de la propia conducta de los familiares. Esta última tuvo más utilidad en las discusiones entre los profesionales acerca del proceso del grupo, que tenían lugar después de cada reunión.

Otras razones aconsejaban también el inicio del grupo, como la necesidad de afrontar la sensación de aislamiento experimentado por muchos familiares de pacientes esquizofrénicos, la posibilidad de que el grupo permitiera a los familiares descargar sus emociones que, de otra manera, recaerían sobre los pacientes, y el hecho de que era un recurso económico. El grupo se constituyó de forma que fuera flexible, tanto respecto a sus miembros como a su asistencia. Los familiares se incluían en el grupo al incorporarse los pacientes al estudio. El grupo se reunía, cada dos semanas, en una habitación grande y confortable del Instituto Psiquiátrico, por espacio de hora y media y los familiares asistían cuando les era posible. Se les animó a que asistiesen los nueve meses del período del estudio, pudiendo continuar después si lo deseaban, y algunos asistieron más de un año. El grupo lo constituían un máximo de siete miembros a la vez e incluía dos profesionales durante la mayor parte del estudio; pero, posteriormente, sólo uno. Nuestra experiencia con el grupo se presenta, con más detalle, en otra parte (Berkowitz et al., 1981). Con el permiso de los familiares se grababa cada sesión para futuros análisis.

\section{Sesiones familiares}

Como el grupo de familiares no era apropiado para tratar la totalidad de los problemas o para un trabajo dinámico y, por otra parte, se excluía de él a los pacientes, pensamos que era necesario complementarlo con sesiones con la familia entera. Consiguientemente, vimos a cada familia, en su propia casa, entre. un mínimo de una y un máximo de veinticinco ocasiones. Las sesiones, que duraban una hora, las dirigían dos profesionales: un psiquiatra y una psicóloga. Cuando el familiar clave era la esposa, veíamos al paciente y a la esposa conjuntamente. Caso de que fueran los padres, veíamos al paciente con sus padres, si era posible, animando también a los hermanos adultos a que participasen.

Al comienzo del estudio el valor de la terapia familiar no estaba bien establecido, particularmente en relación con las familias de los pacientes esquizofrénicos. En consecuencia, adoptamos un enfoque pragmático, guiados por nuestro principal propósito de reducir la EE y/o el contacto social. Las técnicas empleadas para conseguir este fin en las sesiones familiares variaron desde las interpretaciones dinámicas hasta la intervención conductual. Antes de cada sesión discutíamos y formulábamos nuestros objetivos; pero podíamos desviarnos de ellos en caso de que surgiese un acontecimiento importante en el transcurso de la visita. Cada sesión se discutía después y se reflejaba con detalle por escrito.

Además de estos contactos formales, algunos familiares nos telefonearon, a uno u otro de nosotros, en varias ocasiones. Registramos cuidadosamente todos esos contactos para calcular el tiempo total dedicado a cada familia. 


\section{Evaluación}

Fueron analizados los casos de todos los pacientes entre los 16 y los 65 años ingresados en los hospitales Bethlem, Maudsley, Southwestern y el ala norte del de San Pancracio. Si habian vivido de manera continuada con sus familiares los tres meses anteriores a su ingreso, residían a una distancia razonable del hospital correspondiente y su historia reflejaba un trastorno psicótico funcional, se les entrevistaba por medio del Examen del Estado Actual (Present State Examination), PSE (Win et al., 1974). Los datos del PSE se procesaron por el programa Catego y los pacientes diagnosticados de esquizofrenia fueron incluidos en el estudio si, además, habían tenido contacto directo con uno o más familiares durante más de 35 horas semanales. Este extremo se determinó elaborando un baremo de tiempo de una semana típica a partir de la entrevista con el cliente o, en su defecto, con un familiar (Brown et al., 1972).

Si cumplían estos criterios, los pacientes eran incluidos en el estudio, recogiéndose sus datos demográficos y sus antecedentes mediante instrumentos estandarizados. Los familiares de alto contacto directo con el paciente (la esposa o los padres casi siempre) fueron entrevistados con la Entrevista Familiar de Camberwell (Camberwell Family Interview, Vaughn y Leff, 1976b). La valoración de los cuatro principales componentes de la EE: número de críticas, hostilidad, calor e implicación emocional excesiva, fue realizada a través de las grabaciones de las entrevistas. Los familiares con una valoración de 6 o más comentarios críticos, hostilidad en cualquier grado o una puntuación de 3 o más en implicación emocional excesiva, se asignaron al grupo de EE alta. Las demás medidas y procedimientos utilizados se reflejan en el diagrama de la figura 2.

La Entrevista de Conocimiento (Knowledge Interview) se construyó, especialmente, para contrastar los efectos del programa educativo. Constaba de 21 preguntas relacionadas directamente con la información básica facilitada en el programa. Se administró, antes y después del programa, a los familiares del grupo experimental de alta y baja EE y, con el mismo intervalo, a los familiares del grupo de control. La Entrevista de Conocimiento se administró una tercera vez a todos los familiares al final del seguimiento de nueve meses.

Inmediatamente después de la primera Entrevista de Conocimiento se tuvo una entrevista conjunta con el familiar y el paciente en un laboratorio. Ello nos permitió registrar en vídeo la entrevista, durante la cual se recogieron algunas medidas psicofisiológicas de las respuestas del paciente. Los datos de estas entrevistas se presentarán en otra parte.

El siguiente paso fue aplicar el programa educativo a los familiares experimentales de alta y baja EE. Una vez finalizado, se les invitó a incorporarse al grupo de familiares. Para entonces, la mayoría de los pacientes estaban próximos a ser dados de alta del hospital. Se informó a los clínicos responsables del alto riesgo de los pacientes con familiares de alta EE y se les pidió que, en la medida de lo posible, prescribieran neurolépticos de efecto retardado. Debemos indicar que los pacientes siguieron bajo el cuidado de sus clínicos habituales. Nuestras intervenciones terapéuricas con las familias experimentales se realizaron paralelamente con la atención clínica habitual de los pacientes y bajo un contacto directo con el equipo clínico, lo que, en general, constituyó un compromiso viable, aunque no en todos los casos.

Se evaluó el estado clínico de los pacientes inmediatamente antes de ser dados de alta. Si era evidente que el episodio psicótico había remitido 
FIG. 2.-Diagrama de flujo del programa de intervención

Ingreso

Alta

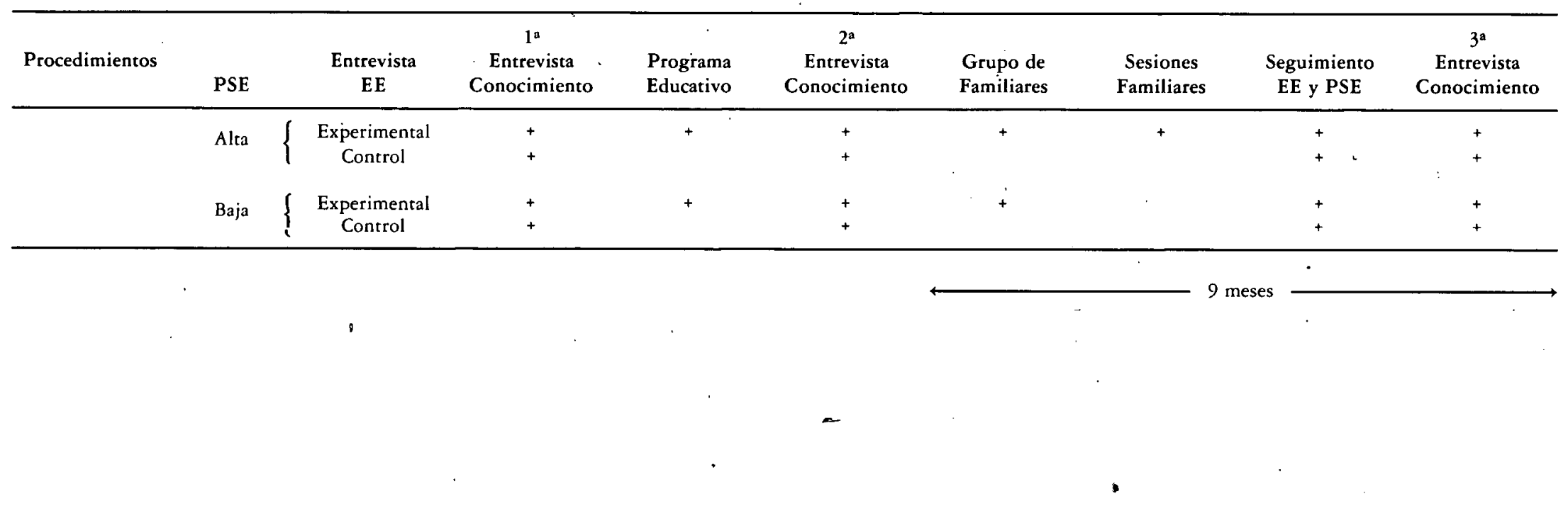


totalmente, se realizaba una evaluación informal. No obstante, en el caso de que persistiesen algunos síntomas psicóticos residuales, se utilizó el PSE completo, que sirvió de línea base para cualquier cambio subsiguiente. Una vez dados de alta del hospital prácticamente todos los pacientes, acudieron de forma regular a clínicas ambulatorias. A los historiales de los casos se adjuntó una nota pidiendo a los clínicos responsables que contactasen con el equipo investigador si existía algún indicio de recaída, ya fuera real o simplemente pudiera sospecharse. En consecuencia, tuvimos la posibilidad de ver a todos los pacientes que sufrieron un recrudecimiento de los síntomas, excepto uno, en un estadio inicial de su recaída. Entonces se realizó un PSE completo y se recogió un historial de los acontecimientos ocurridos en los tres meses anteriores a la recaída (Brown y Birley, 1968).

En esta investigación se definió la recaída como una reaparición de los síntomas esquizofrénicos detectados por medio del PSE, en pacientes que no los presentaban al ser dados de alta (recaida tipo I de Brown et al., 1972). Para el reducido número de pacientes que seguían presentando síntomas de esquizofrenia al ser dados de alta (dos en el grupo experimental y tres en el de control) la recaída se definió como un aumento acusado en el número o intensidad de los síntomas (recaída tipo II). Este último tipo de recaída implica un juicio más subjetivo que el primero, por lo que puede resultar influido por sesgos del entrevistador. Desgraciadamente, no fue posible mantener secreta la condición experimental o de control de cada familia debido a que el equipo de investigación efectuaba tanto la intervención como las valoraciones. Esta deficiencia del procedimiento habría podido remediarse aumentando considerablemente el equipo, pero no tuvimos esa posibilidad. No obstante, se tomaron algunas precauciones para evitar, en lo posible, los sesgos. Las valoraciones clave de EE y del PSE fueron realizadas por los investigadores de mayor experiencia en el uso de las técnicas y que habían sido entrenados para alcanzar un alto nivel de fiabilidad.

La valoración de la EE fue realizada, habitualmente, por dos evaluadores independientes. Los casos de discrepancia, o en los que la valoración era crucial, se sometían a la Dra. Christine Vaughn, quien emitía un juicio a ciegas. Además, una selección representativa de ocho entrevistas de seguimiento fue evaluada a ciegas por el Dr. R. Ghosh, que había seguido un entrenamiento en la valoración de la EE. Su fiabilidad con los jueces se calculó utilizando el coeficiente de correlación producto-momento, resultando ser 0,88 para los comentarios críticos y 0,90 para la implicación emocional excesiva. Este evaluador coincidió con la asignación de las familias a los grupos de alta o baja EE en siete de los ocho casos. En el caso restante valoró a una madre experimental como marginalmente alta en criticismo, en tanto que los dos jueces, independientemente, la consideraron baja.

Las valoraciones del PSE no se hicieron a ciegas en el caso de la recaída tipo I. No obstante, para posibles recaídas del tipo II se recurrió a un evaluador independiente, el Dr. Paul Bebbington, para que efectuara las valoraciones a ciegas. Este no siempre coincidió con la opinión de los clínicos de los pacientes sobre la existencia de una recaída.

\section{Seguimiento}

Todos los pacientes incluidos en el estudio fueron objeto de seguimiento, a lo largo de nueve meses, después de ser dados de alta. Si no habían 
recaído aún, se les administraba el PSE y exploraban sus acontecimientos vitales. Al familiar, o familiares, se les entrevistó nuevamente con la Entrevista Familiar de Camberwell, en la medida de lo posible por el mismo entrevistador que en la primera ocasión, y se valoró la EE. Se recogió de nuevo la distribución del tiempo del paciente. También se obtuvo un historial de la medicación que tomó el paciente durante los nueve meses precedentes.

Se inició, asimismo, un seguimiento de dos años, cuyos resultados se darán a conocer una vez obtenidos.

\section{Sujetos: Características de los pacientes}

En el transcurso de cuatro años, 49 pacientes cumplieron los criterios para ser incluidos en el estudio. El número es tan reducido porque nuestros procedimientos de selección de pacientes de alto riesgo eliminaron a dos de cada tres ingresos con diagnóstico de esquizofrenia. Nueve (18 por $100)$ de las familias rehusaron participar en el estudio. De las restantes, 24 vivían con familiares de $\mathrm{EE}$ alta y participaron en el estudio. Las familias se subdividieron en 12 de control y 12 experimentales, utilizando tablas de números aleatorios. Los familiares que, por medio de la Entrevista Familiar de Camberwell, fueron considerados de EE baja se subdividieron, al azar, en grupos experimental y de control con objeto de valorar los efectos del programa educativo (véase figura 2). Además, a las familias experimentales con EE baja se las animó a que se incorporasen a los grupos de familias, con la principal finalidad terapéutica de identificar y utilizar como modelo sus habilidades de enfrentamiento.

\section{Datos demográficos y biográficos}

Los pacientes experimentales y de control fueron comparados en un gran número de características demográficas y biográficas, como puede verse en la tabla I. Excepto el desempleo, que era mayor en el grupo expe-

TABLA 1

Caracteristicas demográficas y biográficas de los pacientes

\begin{tabular}{|c|c|c|}
\hline & Experimental & Control \\
\hline Número & 12 & 12 \\
\hline 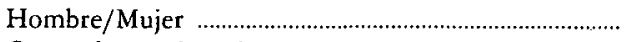 & $6 / 6$ & $6 / 6$ \\
\hline \multicolumn{3}{|l|}{ Grupo de convivencia: } \\
\hline Padres & 6 & 6 \\
\hline Esposa/hermanos & 6 & 6 \\
\hline Mediada de edad & 39 & 30 \\
\hline Educación & 4 & 4 \\
\hline Casado & 6 & 7 \\
\hline Divorciado o separado & 1 & 2 \\
\hline Promedio de hijos . & 0,8 & 1,3 \\
\hline 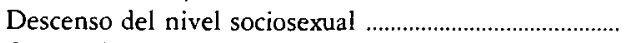 & 3 & 2 \\
\hline 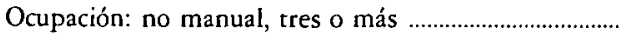 & 6 & 2 \\
\hline $\begin{array}{l}\text { Duración media en el desempleo antes del ingreso } \\
\text { (en meses) }\end{array}$ & 16,5 & $4,8^{*}$ \\
\hline Trabajaban al ingresar & 4 & 7 \\
\hline Personalidad premórbida anormal ........................................ & 8 & 5 \\
\hline 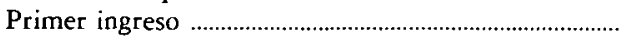 & 4 & 4 \\
\hline Media de ingresos anteriores & 2,3 & 1,2 \\
\hline 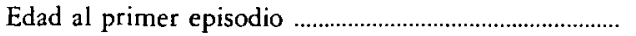 & 33 & 27 \\
\hline
\end{tabular}

* $\mathrm{P}<0,05$ 
rimental, ninguna de las restantes características arrojó diferencias significativas entre los dos grupos, corroborándose la eficacia del procedimiento aleatorio.

\section{Datos clínicos}

Los datos del PSE se procesaron según el programa Catego, obteniendo un perfil de Síndromes y de Categorías Diagnósticas. Se compararon los pacientes experimentales y los de control en cuanto a la presencia, o ausencia, de cada uno de los 38 Síndromes, utilizando el test exacto de Fisher. En ninguno de los Síndromes se obtuvieron diferencias significativas entre los dos grupos, confirmándose, una vez más, la correcta asignación de pacientes al azar. En términos de las Clasificaciones del Catego, 11 pacientes de cada grupo fueron etiquetados $\mathrm{S}+$ (esquizofrenia), en tanto que los pacientes restantes de cada grupo se clasificaron como $\mathrm{P}+(\mathrm{psi}$ cosis paranoide).

\section{Características de los familiares}

En el grupo experimental, seis-pacientes vivían con su esposa y seis con sus padres. En el grupo de control, seis vivían con su cónyuge, cinco con sus padres y uno con su hermana. Algunos pacientes de ambos grupos vivían con uno solo de los padres y en algunas familias uno de los padres mantenía poco contacto con el paciente y, en este caso, no se les entrevistó para la valoración de la EE. Se valoró la EE de un total de 13 familiares del grupo experimental y 13 del grupo de control. La media del número de comentarios críticos y el promedio de las valoraciones de estos pacientes se reflejan en la tabla $\mathrm{I}(a)$ y $b$ ). No se obtuvieron diferencias significativas entre los dos grupos en estas medidas.

\section{RESULTADOS}

\section{Intervención social}

Dos familiares no asistieron, en ningún momento, al grupo. Una esposa asistió la primera vez, en la única ocasión en que no acudió ningún otro familiar. Participó en una sesión individual con el terapeuta y rehusó volver a mantener el contacto. Fue, por otra parte, el único familiar que no recibió el programa educativo. Otro, un marido, era gerente de un negocio que requería su presencia en la hora de reunión del grupo. Los 11 familiares restantes con EE alta participaron en el grupo un promedio de 9,1 veces, asistiendo entre 6 y 21 veces a lo largo de los nueve meses siguientes. El número de familiares presentes en cada sesión variaba de dos a siete, con una moda de cuatro.

Las sesiones de terapia familiar se celebraron todas en los domicilios respectivos. El número de sesiones osciló entre 1 y 25 , con una media de 5,6. No obstante, sólo una familia llegó a las 25 sesiones.

Además de los contactos formales del grupo de familiares y de las sesiones de terapia familiar, se les propuso que telefoneasen a los miembros del equipo siempre que lo consideraran necesario. No abusaron de esta ayuda ya que sólo 10 familiares urilizaron este método de contacto entre 2 y 19 veces, con un promedio de 7,5 veces. 
Aparte de nuestro propio trabajo con los familiares del grupo experimental, el equipo clínico, responsable de la atención a los pacientes, proporcionó alguna ayuda en dos ocasiones. Para una pareja de casados, el asistente social del equipo clínico actuó como coterapeuta con uno de los psicólogos investigadores porque deseaba adquirir experiencia en terapia de pareja. Otro matrimonio siguió una terapia de pareja con un psiquiatra paralelamente a nuestras propias sesiones familiares. Tuvimos dificultades. en mantener una relación estrecha con este terapeuta.

Las experiencias terapéuticas de los familiares de control se estudiaron consultando los historiales de los pacientes, así como preguntando a los familiares en el curso del seguimiento.

\section{Experiencias del tratamiento de los familiares de control}

Ocho casos no recibieron ayuda terapéutica alguna. En dos casos, los propios familiares visitaron a un psiquiatra para recibir tratamiento por síntomas manifiestos. Se descubrió que un familiar, la madre de una muchacha esquizofrénica, sufría esquizofrenia y se le administró tratamiento, como paciente externo, con medicación neuroléptica. Esta madre asistió, en total, 11 veces en el transcurso de los nueve meses; pero el tratamiento se centró en su propia condición psiquiátrica más que en las actitudes hacia su hija. Otro paciente que recibió tratamiento fue un márido con depresión, al que se vio varias veces, como paciente externo, durante dos meses. Otras dos familias recibieron ayuda de asistentes sociales: en una de ellas, en forma de una sesión familiar única celebrada en el noveno mes, después de haber sido dado de alta el paciente, con el propósito de persuadirla para que acudiese al hospital de día. En otra familia, la esposa de un paciente esquizofrénico fue visitada quincenalmente por un asistente social psiquiátrico y manteniendo sesión familiar en el transcurso de los nueve meses. El asistente social escribió: «Mis entrevistas han tenido el objeto de proporcionarle ayuda para compartir su sentimiento de responsabilidad para apoyạr el progreso de su marido.» Le dio algunos consejos sobre cómo hacer frente a la conducta de su esposo, originada por sus delirios. Este es el único caso, entre los 12 controles, en que se prestó una ayuda profesional a los familiares similar a la recibida por las familias experimentales. Es interesante observar que, en este caso control, el nivel de implicación emocional de la esposa aumentó durante el período de seguimiento, a pesar de haber reducido el contacto cara a cara con su marido.

\section{Seguimiento de los familiares}

Todos los familiares del grupo experimental fueron sometidos a una evaluación planificada para el seguimiento a los nueve meses. Hubo una excepción, la madre del último paciente que se incoṛporó al estudio. Cuando finalizó el tiempo de seguimiento con el penúltimo paciente, el último no había sido dado aún de alta del hospital, tras una estancia de casi un año. El estudio se había prolongado ya cinco años, por lo que se decidió presentar los resultados de los 23 pacientes que habían completado el seguimiento, con el fin de evitar un año más de espera. El seguimiento a los dos años está ya bastante avanzado y, cuando finalice, el resultado del seguimiento a los nueve meses del último paciente se incluirá en la presentación de los datos. 
Los familiares de control no fueron tan cooperadores como los experimentales debido, probablemente, a que no les ofrecimos ayuda alguna. De los 13 inicialmente entrevistados, 2 rehusaron una segunda entrevista de EE y uno, un marido, dejó a su esposa y no pudo ser localizado. Por ello, se realizó con éxito el seguimiento en el 77 por 100 de los casos.

En la tabla II a) y b) se muestran el número de comentarios críticos y los valores de implicación emocional excesiva obtenidos en el seguimiento. Es evidente que se produjo una reducción significativa del criticismo en el grupo experimental a lo largo de los nueve meses de la intervención social. Cinco familiares experimentales pasaron de elevado a bajo criticismo y en otros tres se redujo en alguna medida. Sólo tres familiares críticos siguieron virtualmente sin cambio. En contraste, se apreció una ligera reducción no significativa en el número de comentarios críticos hechos por los familiares de control, lo que se explica porque dos familiares (una esposa y una hija) pasaron de alto a bajo criticismo. Como ningún familiar había recibido ayuda terapéutica alguna de los profesionales, esto representa, muy posiblemente, una alteración espontánea en esta actitud.

De estos datos podemos deducir que nuestro paquete de intervenciones sociales obtuvo un éxito considerable en la mejora de actitudes críticas en los familiares experimentales. En cambio, no puede decirse lo mismo en lo que respecta a la implicación emocional excesiva. En los familiares experimentales se produjo un descenso en la valoración media de la implicación emocional excesiva, como puede apreciarse en la tabla II b), aunque sin alcanzar una significación estadística. En el plano individual, dos familiares cambiaron de alta a baja implicación emocional excesiva. Uno de éstos, la única esposa del grupo experimental que mostrạba implicación emocional excesiva, experimentó un cambio espectacular, pasando de una valoración de 4 a 0 . Otros dos bajaron en su valoración, pero sin descender por debajo de 3 , mientras que otro familiar no experimentó cambio alguno. Por el contrario, ninguno de los familiares de control con implicación emocional excesiva disminuyó su valoración por debajo de 3 en esta escala, si bien un familiar cambió de bajo a alto nivel durante el período de seguimiento.

En el seguimiento, el contacto directo había descendido por debajo de las 35 horas semanales en seis familiares experimentales, siendo dos de ellos los padres de un paciente. En un paciente esto obedeció a que había encontrado un trabajo a tiempo completo; en otro a la asistencia al hospital de día; mientras que los tres restantes alteraron la forma de pasar su tiempo libre. Se encontró también un bajo contacto en el seguimiento en tres familias de control, lo que implica que no existe una diferencia significativa con las familias experimentales. Sin embargo, en dos de estas últimas, la manera en que se consiguió este objetivo fue diferente del grupo experimental. El marido de una paciente la dejó dúrante el período de seguimiento, mientras que otro paciente empezó a pasar la mayor parte de su tiempo libre en su habitación. El tercer paciente demostró mayor intẹrés por actividades de tiempo libre, que le mantenían fuera de la casa de sus padres.

Como se ha indicado anteriormente, el objetivo de la intervención social consistía en reducir la EE y/o el contacto directo por debajo de ciertos niveles cruciales. La reducción de EE se consiguió en cinco familias y la reducción del contacto, asimismo, en cinco familias, aunque se produjo algún solapamiento.

Consiguientemente, uno o ambos de nuestros objetivos se vieron cumplidos en 8 de las 11 familias con seguimiento completo hasta la fecha. 
Así, pues, nuestra intervención social tuvo éxito en el 73 por 100 de las familias.

\section{Seguimiento de los pacientes}

En cada grupo se administró, de manera regular, a todos los pacientes, salvo uno, de acuerdo con lo prescrito, inyecciones de neurolépticos de efecto retardado. A un paciente del grupo de control, con un primer episodio de psicosis paranoide, se le prescribió trifluoperacina oral, que tomó puntualmente, mientras que un paciente del grupo experimental no pudo tolerar los efectos colaterales de las inyecciones y pasó a medicación oral, régimen que cumplió meticulosamente.

Durante los nueve meses de seguimiento recayeron siete pacientes, seis de ellos del grupo de control. Todas las recaídas fueron del tipo I. En seis pacientes la recaída fue en forma de un episodio florido similar, si no idéntico, al sufrido anteriormente. Tres de estos pacientes fueron reingresados en el hospital. En el séptimo paciente, del grupo de control, habían desaparecido las alucinaciones auditivas, pero éstas reaparecieron súbitamente.

La proporción de recaídas en el grupo de control fue del 50 por 100 , en tanto que la proporción en la totalidad del grupo experimental fue del 9 por 100 ( $\mathrm{P}$ exacta $=0,041$ ). Por lo tanto, el paquete de intervenciones sociales produjo una reducción significativa de las recaídas esquizofrénicas. La comparación entre la proporción de recaidas del grupo de control con la de los pacientes en cuyas familias conseguimos uno, o ambos, de nuestros objetivos de la intervención social, arroja más luz sobre la especificidad de las intervenciones. De hecho, no recayó ninguno de los pacientes de estas ocho familias, lo que supone una diferencia en la proporción de recaídas, con respecto al grupo de control, de mayor significación (P exac$\mathrm{ta}=0,024)$.

El único paciente experimental que recayó vivía en intenso contacto con su madre, la cual era muy crítica y de implicación emocional excesiva. El paciente tenía, además, dos hándicaps adicionales ya que, aparte de esquizofrénico, presentaba también el síndrome de Turner y era muy bajo de estatura.

En el grupo de control, como ya se ha dicho, dos familiares cambiaron, espontáneamente, de alta EE a baja, entre la valoración inicial y la del seguimiento. En ninguna de estas dos familias recayó el paciente.

\section{DISCUSION}

Concebimos el procedimiento de selección para nuestro estudio con objeto de incluir pacientes esquizofrénicos con alto riesgo de recaída. La eficiencia de nuestro procedimiento fue corroborada por la proporción del 50 por 100 de recaídas en el transcurso de nueve meses en el grupo de control, pese a que seguían siendo tratados regularmente con neurolépticos. La significativa y sustancial baja tasa de recaídas en el grupo experimental evidencia la eficacia terapéutica de nuestro paquete de intervenciones sociales. Sin embargo, no alcanzamos nuestros objetivos de reducir la EE y/o contacto social en todas las familias, sino solamente en tres cuartas partes de ellas. Resulta notable que en este grupo más pequeño de ocho familias no recayó ningún paciente. La única recaída de un paciente experimental ocurrió en una de las tres familias en que no se consiguió disminuir el nivel de EE ni el contacto social. Ello demuestra que la consecución de uno, 
o ambos, de los objetivos señalados es crucial para producir un efecto terapéutico.

La especificidad de nuestro esfuerzo terapéutico, a lo largo de nueve meses, para prevenir totalmente la recaída en la esquizofrenia en un grupo de alto riesgo, evidencia, inequívocamente, tanto la influencia causal de la EE alta de los familiares en la recaída como el efecto protector de un reducido contacto social directo entre paciente y familiar. Nuestra interpretación tentativa de los datos de la figura 1 (Vaughn y Leff, 1976a) cobra ahora un carácter definitivo, tanto más cuanto que las proporciones de recaídas previstas a partir del medio resultan muy aproximadas a las proporciones obtenidas ( 53 por 100 frente al 50 por 100 y 15 por 100 frente al 9 por 100).

Es importante enfatizar que nuestros hallazgos no sólo constituyen evidencia de la influencia causal de la EE de los familiares en el proceso esquizofrénico de los pacientes, sino que confirma la eficacia de una forma de intervención social con estas familias. Hasta ahora esta evidencia era un tanto difusa. Unicamente los estudios de Goldstein et al. (1978) y Hogarty et al. (1974, 1979) satisfacen razonablemente criterios científicos estrictos. Goldstein y sus colegas estudiaron pacientes esquizofrénicos en su primer o segundo ingreso en el hospital siendo, por lo tanto inevitable que su promedio de edad, 23 años, fuera considerablemente inferior al de nuestra muestra, 35 años. Los pacientes fueron dados de alta solamente una o dos semanas después de habérseles administrado inyecciones de flufenacina de efecto retardado. Los sujetos experimentales pasaron por seis sesiones de terapia familiar de crisis con la finalidad principal de ayudar al paciente y a los otros significativos y a servirse de los acontecimientos de la psicosis para adaptarse a la enfermedad. A los seis meses de seguimiento no había recaído ninguno de los pacientes que tomaban medicación y seguían terapia familiar, mientras que la proporción de recaídas de los que sólo tomaban medicación se elevó al 18 por $100(\mathrm{P}=0,04)$. La interpretación de estos resultados queda ligeramente oscurecida por el hecho de que la terapia recibida por ambos grupos no se estandarizó después de las seis primeras semanas del período de la prueba.

En los dos estudios de Hogarty y sus colegas se añadieron terapias sociales a la administración de medicamentos neurolépticos en un diseño controlado. Sus pacientes eran más similares a los nuestros que los de Goldstein, siendo principalmente crónicos y con una media de tres ingresos en el hospital. En el primer estudio la terapia consistía en una combinación de trabajo social intensivo y consejos de orientación profesional, mientras que el segundo comprendía trabajo social intensivo del individuo y su familia. En ninguno dè estos estudios la intervención social redujo significativamente la proporción de recaídas si se simultaneaba con la administración de medicamentos. No obstante, pareció conferir una ventaja a los pacientes en el segundo año de seguimiento, no dándose ninguna recaída, en este periodo, entre los pacientes que recibían terapia social y medicación en ambos estudios. Así nuestros resultados se aproximan más a los de Goldstein et al., si bien nosotros estudiamos una población más crónica en remisión y no tanto trastornos psicóticos agudos y, además, seleccionamos a sujetos que representaban a un grupo de alto riesgo. Esta diferencia se evidencia comparando la proporción de recaídas de nuestro grupo de control a lo largo de nueve meses, 50 por 100, con la de los pacientes de control de Goldstein en el transcurso de seis meses, 18 por 100.

Nuestros hallazgos suponen importantes implicaciones, tanto de carácter teórico como práctico. En el aspecto teórico, una vez ha quedado fir- 
memente establecido el papel de las actitudes emocionales de los familiares como causa de recaídas en la esquizofrenia, se plantea la cuestión de si operan de igual manera antes de la aparición del primer episodio. Lógicamente es inadmisible extrapolar nuestros hallazgos en el tiempo a lo que sucedió antes de la primera manifestación del trastorno, dado que éste puede alterar dramáticamente las actitudes de los familiares. Sin embargo, existe evidencia de la relación entre una actitud emocional, que consideramos influyente, con una protección materna excesiva antes del inicio de la esquizofrenia. Tal evidencia, deducida de los estudios de clínicos infantiles, fue revisada por Hirsch y Leff (1975), quienes concluyeron que las actitudes de sobreprotección eran más frecuentes en madres cuyos hijos sufrieron, más tarde, esquizofrenia, que en las madres de los niños de control. Existe una clara relación entre, esta sobreprotección previa y un aspecto de la implicación emocional excesiva, medida en los estudios de EE. Esto sugiere que el componente de sobreprotección de la implicación emocional se desarrolla muy tempranamente en la vida del niño y, de hecho, tenẹmos evidencia anecdótica de ello en los relatos de varias de nuestras familias. Es probable que la naturaleza estable de este componente de implicación emocional materna influyera en el nivel inferior de éxito que obtuvimos al intentar alterar esta actitud, en comparación con nuestro efecto sobre el criticismo. La única desaparición espectacular de implicación emocional no ocurrió a una madre sino a una esposa, quien necesariamente había mantenido esta actitud durante un menor período de tiempo.

Otra consideración teórica es el mecanismo por el cual las actitudes de los familiares producen una reaparición o exacerbación de síntomas esquizofrénicos en los pacientes. Estudios anteriores han establecido que los efectos de familiares con EE alta sobre los pacientes pueden detectarse en las respuestas autonómicas de los pacientes (Tarrier et al., 1979; Sturgeon et al., 1981). Se ha propuesto una hipótesis de activación para explicar estos hallazgos y recientemente se ha empezado a trabajar empleando este paradigma para estudiar la psicofisiología cortical.

Se suscitan algunos problemas prácticos, el primero de los cuales se refiere a nuestros fracasos en el grupo experimental. Considerándolo retrospectivamente, ¿hubiéramos podido, quizá, obtener mejores resultados con las tres familias en cuestión, teniendo en cuenta nuestra inexperiencia con este tipo de familias cuando empezamos nuestro estudio? Para una de las familias la respuesta es, posiblemente, sí. Se trataba de un matrimonio en el que el esposo era el paciente. Recibieron terapia de pareja de un psiquiatra clínico paralelamente a nuestras sesiones de terapia familiar. Es posible que esta diversificación del esfuerzo debilitara la potencialidad de los dos grupos de terapeutas y que podría haberse conseguido mejor resultado con un solo grupo.

Las otras dos familias presentaban algunas características en común: ambas tenían múltiples problemas, en ambas el paciente era hija única y el familiar clave era la madre, excesivamente crítica y, a la vez, excesivamente implicada emocionalmente. En una familia, a la que nos hemos referido ya, la paciente sufría el síndrome de Turner, además de esquizofrenia, y la madre había mostrado excesiva implicación emocional casi desde el nacimiento. En la otra, la madre era italiana y no hablaba bien el inglés $y$, retrospectivamente, ponemos en duda si llegó a captar mucho de lo que tratamos en los grupos y las sesiones familiares.

En la primera de estas dos familias, el paciente recayó sólo dos meses después de estar en casa y antes de que su madre hubiera asistido a los grupos familiares. Se trató de un retraso inusual, pudiendo ser síntoma de 
la resistencia de la madre a la terapia. Por nuestra parte, proseguimos trabajando con la familia siguiendo la recaída del paciente, y después de un año, o más, hay indicios de cambio en las actitudes de la madre, extremo que será evaluado a los dos años de seguimiento. La segunda de estas dos familias, cuya madre era italiana, creemos que, probablemente, se habría resistido a los esfuerzos del terapeuta más experimentado. Sin embargo, quizá alguien que hablase inglés e italiano habría podido romper la barrera de la comunicación.

Los otros dos problemas prácticos están estrechamente relacionados: ¿qué elemento del paquete terapéutico fue más eficaz y qué podemos aportar a los profesionales que intentan trabajar con familias de pacientes esquizofrénicos? No podemos contestar totalmente a la primera pregunta porque no evaluamos los elementos individuales de nuestro conjunto de estrategias, aparte del programa educativo. Careciendo de esta información, pensamos que el grupo de familiares y las sesiones familiares revisten igual importancia. l.I trabajo con las familias requiere un entrenamiento más prolongado que el que se necesita para los grupos de familiares; sin embargo, es preciso considerar que, cuando empezamos, teníamos poca experiencia en cualquier forma de terapia con familias de pacientes esquizofrénicos y, pese a ello, alcanzamos una elevada proporción de éxito en la consecución de nuestros objetivos. Creemos que parte de la fórmula para el éxito es establecer, desde el principio, unos objetivos claros y todo aquel que se integre en esta área de trabajo clínico debe proponerse reducir el contacto directo y las actitudes críticas y excesiva implicación emocional en los familiares.

Al clínico se le plantea el problema de decidir qué familias necesitan este tipo de tratamiento. Desgraciadamente, el entrenamiento que exige la valoración de EE es un proceso largo y arduo, de manera que la Entrevista Familiar de Camberwell (Camberwell Family Interview) no puede ser incorporada de manera habitual en la evaluación clínica. No obstante, muchas familias con EE alta son fácilmente identificadas por los clínicos, incluso por los no familiarizados con los conceptos de criticismo e implicación emocional excesiva. A las familias con niveles límite en estas escalas, probablemente no se las identificará en la práctica clínica: pero el clínico debe estar alerta respecto a la posibilidad de que los familiares sean de EE alta si los pacientes que viven con ellos necesitan frecuentes reingresos a pesar de la medicación profiláctica.

La determinación del grado de contacto directo es un procedimiento simple que no exige especial entrenamiento y puede ser fácilmente incluido como uno de los datos a tomar en el historial. A la hora de elegir entre las estrategias para intentar reducir el contacto directo o reducir la EE, puede aceptarse que la primera es la opción más fácil y que exige menos tiempo. En realidad, nosotros empezamos el estudio con esta suposición explícita. Sin embargo, nos encontramos con que algunas familias eran reacias a pasar menos tiempo juntas y, no obstante, respondieron a nuestros intentos de reducir la EE. En otras familias, la reducción del contacto sólo fue posible cuando se relajaron los lazos emocionales que las unían. A la vista de los resultados, tuvimos igualmente éxito en la consecución de ambos objerivos, por lo que recomendamos su adopción conjunta.

Desde el comienzo, consideramos la necesidad de mantener nuestra intervención dentro de la esfera de la práctica clínica cotidiana. Consideramos de tanta importancia práctica el resultado de nuestro estudio que contemplamos en nuestra próxima tarea la compilación de un programa de entrenamiento que pondrá los medios necesarios al alcance de los asistentes sociales, psicólogos clínicos y ATS psiquiátricos. 


\section{Referencias}

BerkowitZ, R.; KUIPERS, L.; EBERLEIN-Fries, R., LEFF, J. «Lovering expressed emotion in relatives of schizophrenics», en New Developments in Interventions with Families of Schizophrenics (Ed. M. S. Goldstein), Londres: Jossey-Bass, 1981.

Brown, G. W., y BIRLEY, J. L. T. «Crises and life changes and the onset of schizophrenia». Journal of Health and Social Behaviour, 1968, 9, 203-214.

BROWN, G. W.; BIRLEY, J. L. T., y WING, J. K. «Influence of family life on the course of schizophrenic disorders: a replication». British Journal of Psychiatry, 1972, 121, 241-258.

Brown, G. W.: MONCK, E. M.; Carstairs, G. M., y WING, J. K. «Influence of family life on the course of schizophrenic illness». British Journal of Preventive and Social Medicine, 1962, 16, 55-68.

CReER, C., y WING, J. «Living with a schizophrenic patient». Bristish Journal of Hospital Medicine, 1975, 14, 73-82.

Goldstein, M. J.; Rodnick, E. H.; Evans, J. R.; May, P. R. A., y SteinberG, M. R. «Drug and family therapy in the aftercare of acute schizophrenics». Archives of General Psycbiatry, 1978, 35, 1169-1177.

HiRSCH, S.R., y LefF, J. P. Abnormalities in parents of schizopbrenics. Maudsley Monograph, $\mathrm{n}^{0} 22$. Londres; Oxford University Press, 1975.

HOGARTY, G. E., y GOLDBERG, S.C. «Drug and sociotherapy in the aftercare of schizophrenic patients. One-year relapse rates». Archives of General Psychiatry, 1973, 28, 54-64.

HogarTy, G.E.; Goldberg, S.C.; SCHOOlER, N.R., y UlRich, R.F. «Drug and sociotherapy in the aftercare of schizophrenic patients. II. Two years relapse rates». Arcbives of General Psychiatry, 1974, 31, $603-608$.

Hogarty, G. E.; Schooler, N. R.; Ulrich, R. F.; Mussare, F.; Ferro, P., y Herron, E. «Fluphenazine and social therapy in the aftercare of schizophrenic patients». Archives of General Psycbiatry, 1979, 36, 1283-1294.

LEFF, J. P. «Schizophrenia and sensitivity to the family environment». Schizophrenia Bulletin, 1976, 2, 566-574.

- «Clinical en methodological problems in interaction studies», en Epidemiological Impact of Psychotropic Drugs (Eds. G. Tognoni, C. Bellantuono y M. Lader). Amsterdam: Elsevier, 1981.

LEFF, J. P., y VAUGHN, C. «The role of maintenance therapy and relatives expressed emotion in relapse of schizophrenia: a two year follow-up» British Journal of Psycbiatry, 1981, 139, 102-104.

LEFF, J. P., WING, J. K. «Trial of maintenance therapy in schizophrenia». Bristisb Medical Journal, 1971, iii, 599-604.

Oford, J., y EDwards, G. Alcoholism. Maudsley Monograph ne 26. Londres: Oxford University Press, 1977.

PRIESTLEY, D. «Schizophrenia and the family: Part II», en Community Care of the Mentally Disabled (Eds. J. K. Wing y R. Olsen). Londres: Oxford University Press, 1979.

STURGEON, D.; KUIPERS, L.; BerkowiTZ, R.; TuRPIN, G., y LefF, J. «Psycophysiological responses of schizophrenic patients to high and low expressed emotion relatives». British Journal of Psychiatry, 1981, 138, 40-45.

TARRIER, N.; VAUGHN, C.E.; LADER, M. H., y LeFF, J. P. «Bodily reaction to people and events in schizophrenia». Archives of General Psychiatry, 1979, 36, 311-315.

VAUGHN, C. E., y LEFF, J. P. "The influence of family and social factors on the course of psychiatric illness. A comparison of schizophrenic and depressed neurotic patients" British Journal of Psychiatry, 1976a, 129, 125-137.

VAUGHN, C. E.; LEFF, J. P. «The measurement of expressed emotion in families of psychiatric patients». Bristish Journal of Social and Clinical Psychology, 1976b, 15, 157-165.

WING, J. K.; COOPER, J. E., y SARTORIUS, N. Measurement and classification of Psychiatric Symptoms. Cambridge: Cambridge University Press, 1974. 